\title{
Médiévales
}

Langues, Textes, Histoire

62 | printemps 2012

Hagiographie et réforme dans l'Occident latin

\section{Pillages et butins dans la représentation du pouvoir à l'époque carolingienne}

Plunder and Booties in Carolingian Representation of Power

\section{Rodolphe Keller}

\section{(2) OpenEdition \\ Journals}

Édition électronique

URL : https://journals.openedition.org/medievales/6711

DOI : 10.4000/medievales.6711

ISSN : $1777-5892$

Éditeur

Presses universitaires de Vincennes

Édition imprimée

Date de publication : 2 juin 2012

Pagination : 135-152

ISBN : 978-2-84292-346-4

ISSN : 0751-2708

\section{Référence électronique}

Rodolphe Keller, «Pillages et butins dans la représentation du pouvoir à l'époque carolingienne », Médiévales [En ligne], 62 I printemps 2012, mis en ligne le 05 juillet 2012, consulté le 22 avril 2022. URL : http://journals.openedition.org/medievales/6711; DOI : https://doi.org/10.4000/medievales. 6711 
Rodolphe KELLER

\section{PILLAGES ET BUTINS \\ DANS LA REPRÉSENTATION DU POUVOIR À L'ÉPOQUE CAROLINGIENNE}

En 1985, dans un article novateur sur les pillages et tributs à l'époque carolingienne, Timothy Reuter rappelait que, pour une grande partie de l'Europe des VIII ${ }^{e}$ et IX $\mathrm{IX}^{\mathrm{e}}$ siècles, «c'étaient les Francs qui étaient les Vikings ${ }^{1}{ }^{1}$. Notre vision du haut Moyen Âge reste en effet largement marquée par quelques grandes images, comme celles de l'«expansion carolingienne» ou des «invasions scandinaves », qui ordonnent encore - en filigrane - notre approche de la période. Ces représentations sont, dans une certaine mesure, le reflet du tableau donné par les sources contemporaines elles-mêmes, comme les annales du IX ${ }^{\mathrm{e}}$ siècle, qui égrènent, année après année, les incursions de Vikings païens, barbares et pilleurs, face à des Francs qui sont présentés tantôt comme conquérants, tantôt comme victimes des déprédations. Cette opposition demeure implicitement présente dans l'historiographie actuelle, certaines sociétés étant traditionnellement évoquées surtout dans leur dimension prédatrice.

Depuis quelques années, la recherche a fortement nuancé ce tableau. Pierre Bauduin a récemment illustré la complexité des relations entre Francs et Scandinaves, par une analyse très fine des stratégies permettant la coexistence entre les groupes ${ }^{2}$. Une autre approche possible consiste à repenser la prédation et sa place dans les sociétés du haut Moyen Âge. Les sources sont nombreuses à évoquer des armées franques s'emparant de riches butins. Timothy Reuter a souligné l'importance de pratiques comme le pillage ou le prélèvement tributaire

1. T. ReUTER, «Plunder and tribute in the Carolingian empire», dans Transactions of the Royal Historical Society, 5/35 (1985), p. 91.

2. P. Bauduin, Le Monde franc et les Vikings ( $\left(\mathrm{VII}^{e}-\mathrm{X}^{e}\right.$ siècle), Paris, 2009. 
dans les dynamiques politiques et sociales de l'empire carolingien ${ }^{3}$. Or, si la prédation comporte un caractère systémique dans la société franque, on doit poser la question de sa place au sein des systèmes de valeurs concernés et, plus particulièrement, dans la représentation du pouvoir.

Cela soulève le problème de la légitimité de la prédation. Il faut alors préciser les enjeux du problème. Il est aisé de constater que les transferts contraints de biens ou de personnes exercés par la violence sont considérés comme légitimes, s'ils sont pratiqués contre des groupes extérieurs identifiés a priori comme ennemis, par exemple contre les Saxons païens. Cependant, le fait que le pillage est considéré comme légitime en soi dans le cadre d'une guerre, n'implique pas nécessairement qu'il soit légitime de mener une guerre afin de mener des pillages. En d'autres termes, on doit s'interroger sur la légitimité de la guerre prédatrice, conçue comme l'une des modalités d'exercice du pouvoir, et plus particulièrement sur son articulation avec l'idéologie royale carolingienne.

\section{Légitimité de la prédation et idéologie du pouvoir}

Partons d'un cas d'école particulièrement éclairant, sur lequel nous reviendrons à plusieurs reprises: la guerre menée par les Carolingiens contre les Avars dans les années 790. C'est précisément au cours de ce conflit que se produit un des pillages les mieux documentés, parmi ceux effectués par les armées carolingiennes. Un bref rappel du contexte est ici utile. Les Avars sont, à l'origine, une population nomade d'Asie centrale ${ }^{4}$. Au cours du vi siècle, à la suite de leurs migrations, ils se sont installés dans la plaine pannonienne (moyen Danube), où ils ont imposé leur domination aux populations slaves locales, tout en exigeant de Byzance le versement de lourds tributs. À partir de la fin du vII' siècle, l'empire avar subit un certain déclin. Durant la seconde moitié du viII ${ }^{\mathrm{e}}$ siècle, ce sont les affaires bavaroises qui mènent à l'affrontement avec le pouvoir carolingien. En effet, en 788, le duc de Bavière Tassilon, qui s'était allié avec les Avars, est évincé par Charlemagne et la Bavière est placée sous contrôle direct du roi franc, par l'institution d'un praefectus. Rapidement, se pose un problème de définition des frontières, qui déclenche un premier affrontement en 791: une

3. Philip Grierson et Georges Duby avaient, les premiers, mis en avant l'importance de ces pratiques dans les transferts de biens au haut Moyen Âge, sans approfondir la question. Cf. P. H. GRIERSON, «Commerce in the dark Ages : a critique of the evidence», dans Transactions of the Royal Historical Society, 5/9 (1959), p. 123-140, et G. Duby, Guerriers et paysans (VII -XII siècle). Premier essor de l'économie européenne, Paris, 1973, p. 60-69. Timothy Reuter a proposé la première analyse complète de la prédation dans le fonctionnement des pouvoirs carolingiens. Cf. T. REUTER, «Plunder...», p. 75-94.

4. Sur les Avars, cf. W. Poнl, Die Awaren : ein Steppenvolk in Mitteleuropa. 567-822 n. Chr., Munich, 1988. 
expédition carolingienne mène alors à la formation d'une marche danubienne. Elle est suivie par des préparatifs en vue de nouvelles interventions. En 795 et 796, deux nouvelles expéditions sont menées: d'abord par Éric, duc de Frioul, à la fin de 795, et ensuite par le fils de Charlemagne, Pépin, roi d'Italie, pendant l'été 796. Ces interventions provoquent la chute de l'empire et le pillage du hring, le siège du pouvoir avar, dont le trésor est envoyé à la cour d'Aix-laChapelle $^{5}$. Les motifs à l'origine de ces affrontements sont relativement bien connus : ils ont été analysés par Josef Deér, qui opère à ce propos une distinction, selon les différentes phases du conflit. La question frontalière serait au centre de l'expédition de 791, alors qu'en 795 et 796 (cf. carte page suivante), les deux expéditions auraient pour objectif principal la capture du trésor avar 6 .

Nous devons alors nous interroger sur le traitement de l'événement par les contemporains. Rappelons d'abord que depuis l'accession des Pippinides au pouvoir royal, au milieu du $\mathrm{VIII}^{\mathrm{e}}$ siècle, les contours de l'idéologie royale ont, à certains égards, évolué, principalement en raison de l'importance croissante du clergé dans le fonctionnement du pouvoir ${ }^{7}$. La réflexion sur les prérogatives du roi élaborée par les lettrés carolingiens comporte des éléments sur la dimension

5. Sur les campagnes avares: J. DeÉR, «Karl der Große und der Untergang des Awarenreiches», dans H. Beumann éd., Karl der Grosse, vol. I: Persönlichkeit und Geschichte, Düsseldorf, 1965, p. 719-791; W. PoHL, Die Awaren..., p. 312-323 et ID., Die Awarenkriege Karls des Großen, 788803, Vienne, 1988; H. WOLFRAM, Grenzen und Räume. Geschichte Österreichs vor seiner Entstehung, Vienne, 1995,p. 233-240. Les Annales royales et les Annales de Lorsch sont les principales sources sur les événements de 795/796. Cf. Annales Regni Francorum, a. 796: «Sed et Heiricus dux Foroiulensis missis hominibus suis cum Wonomyro Sclavo in Pannonias hringum gentis Avarorum longis retro temporibus quietum, civili bello fatigatis inter se principibus spoliavit, - chagan sive iuguro intestina clade addictis et a suis occisis - thesaurum priscorum regum multa seculorum prolixitate collectum domno regi Carolo ad Aquis palatium misit. [...] Rex collectis exercitibus suis Saxoniam ingressus est, filium suum Pippinum regem Italiae in Pannonias cum exercitu misso. Cuius legationes ad eum in eadem Saxonia venerunt, una, quae dixit occurrisse ei kagan cum ceteris optimatibus, quem sibi Avares post interfectionem priorum constituerunt; altera, quae dixit Pippino cum exercitu suo in hringo sedere. Et domnus rex [...] in Aquis palatio filium suum Pippinum e Pannonia redeuntem et partem thesauri, quae remanserat, adducentem laetus aspexit.» (éd. F. KuRZE, MGH SS Rer. Germ. in us. sch. 6, Hanovre, 1895, p. 98-100); on peut comparer ce récit à celui des Annales Laureshamenses, a. 795 et 796, éd. G. H. PERTZ, MGH SS 1, Leipzig, 1826, p. 36. Contrairement à ce que suggèrent les Annales royales, la première expédition a lieu vers la fin de l'année 795: cf. W. PoHL, Die Awaren..., p. 463-464, n. 85, sur la base de la démonstration de S. ABEL/B. von Simson, Jahrbücher des fränkischen Reiches unter Karl dem Großen, t. 2: 789-814, Leipzig, 1883, p. 98.

6. J. DeÉR, «Karl der Große...», p. 784-791. Tout porte en effet à croire que ces expéditions ne visaient en aucun cas une quelconque conquête de l'empire, car celui-ci ne fait l'objet d'aucune intégration politique. On n'observe pas davantage un véritable effort de christianisation, malgré les vœux répétés d'Alcuin.

7. J. L. Nelson, «Kingship and royal government», dans R. McKitterick éd., The New Cambridge Medieval History, t. II : c. 700-c. 900, Cambridge, 1995, p. 383-430 et Y. SASSIER, Royauté et idéologie, Paris, 2003, p. 116-180. 


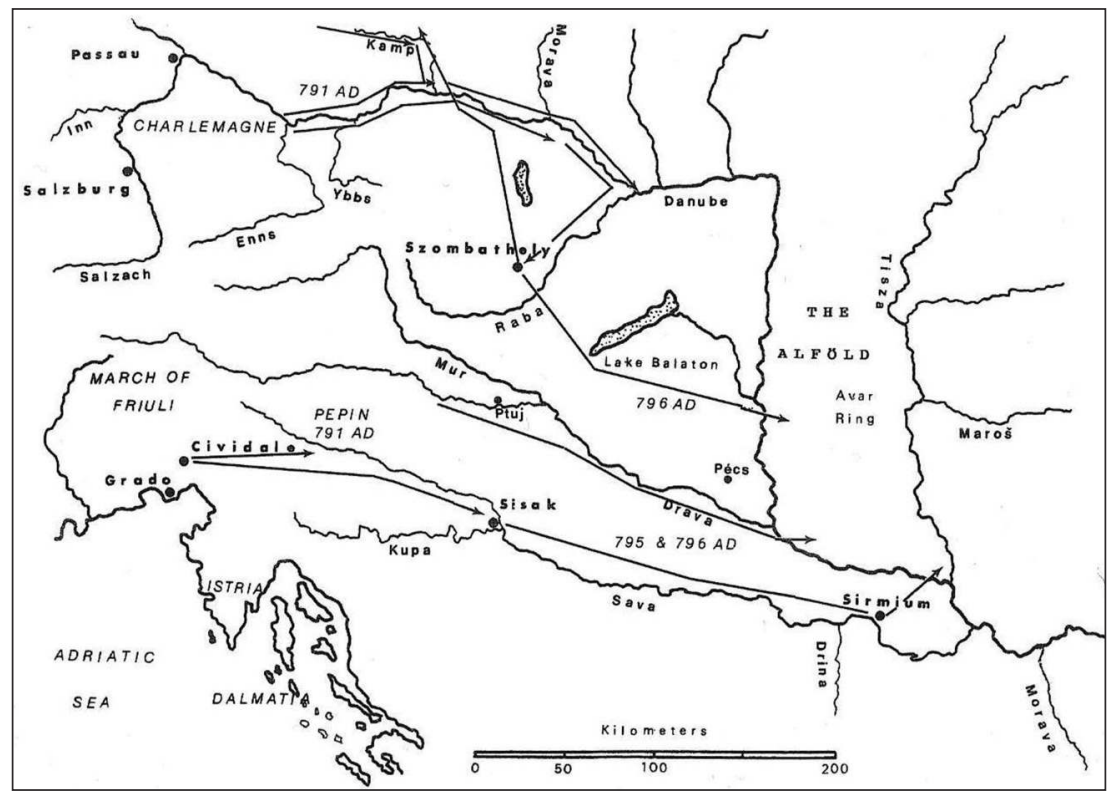

Tracé des expéditions franques contre les Avars, en 791, 795 et 796

(D'après Charles Bowlus, Franks, Moravians, and Magyars.

The Struggle for the Middle Danube, 788-907, Philadelphie, 1995, p. 50)

guerrière de la royauté. La notion augustinienne de «guerre juste» (bellum iustum), implicite chez Alcuin, exprimée plus clairement dans les écrits de Jonas d'Orléans ou plus tard, d'Hincmar de Reims, représente un effort pour justifier la guerre dans le gouvernement d'un prince chrétien, en même temps qu'elle en limite la pratique légitime à certains cas particuliers, comme la défense du populus Christianorum ou la diffusion de la chrétienté ${ }^{8}$. Par ailleurs, la documentation d'époque carolingienne montre l'importance croissante d'une conception pacifique de la royauté. Le roi - rex pacificus - compte, parmi ses attributions, celle de faire régner la paix, d'autant plus que «ce devoir n'était pas exclusivement de nature politique, il avait également acquis un fondement religieux ${ }^{9} »$. Cet aspect se trouve renforcé par le développement de l'idéologie impériale carolingienne. La formule d'acclamation de Charlemagne, lors du

8. K.-G. CRAm, Iudicium Belli. Zum Rechtscharakter des Krieges im deutschen Mittelalter, Berlin, 1955 ; T. ScharfF, Die Kämpfe der Herrscher und der Heiligen. Krieg und historische Erinnerung in der Karolingerzeit, Darmstadt, 2002.

9. F.-L. Ganshof, «La «paix » au très haut Moyen Âge», dans La Paix, Recueils de la société Jean Bodin 14, Bruxelles, 1961, p. 402. 
couronnement du 25 décembre 800, comporte le qualificatif de pacificus, qui doit être compris au sens de «faiseur de paix ${ }^{10}$.

Ces différents aspects de l'idéologie royale et impériale tendraient à disqualifier l'idée d'une légitimité de la guerre prédatrice. On les retrouve à plusieurs reprises, dans l'historiographie carolingienne, comme arguments de justification des conflits menés par les Francs, et précisément dans le cas de la guerre contre les Avars. Joseph Deér, dans son analyse de l'historiographie carolingienne concernant ce conflit, a constaté que les arguments des sources contemporaines s'inscrivent souvent dans la logique de la guerre légitime d'inspiration augustinienne. D'après les Annales royales, la première expédition de 791 est menée «en raison du mal, grand et intolérable, que les Avars ont fait contre la sainte Église et le peuple chrétien ${ }^{11}{ }$. Concernant les événements de 796, plusieurs sources développent le même type de discours. Le Carmen de Pippini regis victoria Avarica, écrit peu après les événements par un auteur anonyme, mais vraisemblablement proche de la cour, évoque les maux multiples perpétrés par les Avars contre les églises. Il célèbre le « roi catholique, ceint de la vertu divine » et rend grâce à Dieu pour la victoire contre les «peuples païens ${ }^{12}$ ». Alcuin se félicite également de cette victoire qui doit permettre leur conversion ${ }^{13}$.

Cependant, si on tient compte de l'ensemble de la production écrite évoquant ces événements, il en ressort une image plus contrastée, qui permet de proposer une interprétation plus complexe de la place de la guerre dans l'ensemble des représentations qui concernent la royauté. En effet, il serait réducteur de voir dans la présentation du conflit comme une guerre juste, un procédé visant à dissimuler des motivations géopolitiques ou matérielles inavouables. Elle n'est en effet pas exclusive de l'évocation parfaitement assumée de motifs plus pragmatiques, par le même entourage carolingien. Les deux ordres d'idées apparaissent comme parfaitement complémentaires. En effet, le pillage du hring est mentionné par de si nombreuses sources, qu'il s'agit de l'un des événements les mieux documentés de l'époque carolingienne. Certaines sources proviennent de l'entourage immédiat du pouvoir, comme les Annales royales ou les Annales de Lorsch. L'événement est également évoqué par un grand nombre d'annales mineures ${ }^{14}$, ainsi que par des biographies, des poèmes, des lettres. Certaines sources sont contemporaines des événements, d'autres plus tardives.

10. Annales Regni Francorum, a. 801, p. 112.

11. Annales Regni Francorum, a. 791, p. 88.

12. Carmen de Pippini regis victoria Avarica, éd. O. Holder-EgGer et G. WaITz, Einhardi Vita Karoli, MGH SS Rer. Germ. in us. schol. 25, Hanovre, 1911, p. 42.

13. Alcuini epistolae, éd. E. DüMmLER, MGH Epp. 4, Berlin, 1895, nº 99, p. 143-144.

14. Par exemple, Annales Guelferbytani, a. 795 et 796, éd. G. H. Pertz, MGH SS 1, Leipzig, 1826, p. 45; Annales Alamannici, a. 796 et 797, éd. G. H. Pertz, MGH SS 1, Leipzig, 1826, 
Aussi bien dans les Annales royales que dans les Annales de Lorsch, c'est-à-dire dans les principales sources de ces événements, s'il est vrai que le conflit de 791 est justifié par la nécessité de la défense du peuple chrétien, ce système argumentatif est en revanche totalement absent du récit concernant 795 et $796^{15}$. Aucun motif particulier n'est donné pour les expéditions d'Éric de Frioul et de Pépin d'Italie. Aucune mention n'est faite d'anciens pillages avars, de destructions d'églises, de leur perfidie, ni même de leur paganisme. Ces textes insistent en revanche sur le pillage du hring ${ }^{16}$. La même constatation peut être faite en ce qui concerne la présentation des événements par Éginhard, auquel nous devons l'unique biographie de Charlemagne écrite par l'un de ses contemporains, la Vita Karoli. Éginhard n'inscrit pas davantage le conflit avar dans une logique de guerre juste. Il ne donne aucun motif à ce conflit, ce qui est d'autant plus étonnant qu'il le présente comme le plus important du règne de Charlemagne, à l'exception de la guerre contre les Saxons ${ }^{17}$. En revanche, il évoque longuement les richesses prises en Pannonie, en précisant qu'il s'agit du plus gros butin jamais obtenu dans une guerre par les Francs, à tel point qu'en comparaison, ceux-ci semblaient auparavant «presque pauvres». Il précise tout de même que les Francs enlevèrent justement aux Avars ce que ces derniers avaient enlevé aux autres peuples ${ }^{18}$. Sous certaines conditions donc, non seulement l'obtention d'un butin permet de légitimer le fait militaire, mais en plus, cela semble constituer un élément important, valorisant, dans le modèle du prince idéal dont Éginhard se fait l'écho. Les descriptions d'annales mineures vont dans le même sens. Ces sources sont constituées de brèves mentions, d'autant plus significatives qu'elles sont très lapidaires. L'entrée pour 796 des Annales Guelferbytani indique que «Charles se rendit à nouveau en Saxe, il pilla cette terre, et en livra la plus grande partie au feu. Il envoya Pépin en Pannonie contre le Hrinc et il emmena de là un grand trésor à Aix, et ici il hiverna; et, une fois encore, les Avars firent défection ${ }^{19}$.» Ces formules stéréotypées sont fréquentes et révèlent les procédés de hiérarchisation des événements par les annalistes, qui

p. 48; Annales Sancti Amandi, a. 796, éd. G. H. PerTz, MGH SS 1, Leipzig, 1826, p. 14; Annales Nordhumbrani, a. 795, éd. R. Pauli, MGH SS 13, Hanovre, 1881, p. 155.

15. Cf. supra, note 5 .

16. Cf. supra, note 5 .

17. ÉGinhard, Vita Karoli, c. 13, éd. O. Holder-EgGER et G. Waitz, MGH SS Rer. Germ. in us. sch. 25, Hanovre, 1911, p. 15.

18. Ibid.,p. 16: «Omnis pecunia et congesti ex longo tempore thesauri direpti sunt. Neque ullum bellum contra Francos exortum humana potest memoria recordari, quo illi magis ditati et opibus aucti sint. Quippe cum usque in id temporis poene pauperes viderentur, tantum auri et argenti in regia repertum, tot spolia pretiosa in proeliis sublata, ut merito credi possit hoc Francos Hunis iuste eripuisse, quod Huni prius aliis gentibus iniuste eripuerunt.»

19. Cf. supra, note 14 . 
ne retiennent que quelques éléments-clés qu'ils jugent importants - souvent les pillages ou la capture d'un trésor. Leur attention soutenue aux faits de prédation suggère la permanence d'une conception de la royauté qui remonte, au moins, aux Mérovingiens.

La royauté franque à l'époque mérovingienne se caractérise par une dimension sacrale et magique, qui résulte d'un ensemble de représentations complexes renvoyant à une structure symbolique du $\operatorname{cosmos}^{20}$. Parmi les aspects symboliques de la royauté, on peut retenir deux éléments qui sont présents dès les premiers temps de la royauté mérovingienne. D'une part, le roi est garant de la fertilité. Cette royauté nourricière s'exprime par des rites de fertilité comme les parcours rituels sur des bœufs dont Éginhard se moque plus tard, dans son récit sur les «rois fainéants ${ }^{21}$. D'autre part, la royauté mérovingienne comporte une forte dimension guerrière. Il faut noter que ces deux aspects de la royauté expriment, de manière cohérente, l'idée d'une royauté garante de la prospérité du peuple, car la fonction guerrière semble, dans ce système de représentations, avoir une forte dimension économique, étant entendue aussi comme mode de prélèvement de ressources.

Cet aspect du roi guerrier est bien illustré dans le symbolisme de la chasse, qui donne lieu à des rituels royaux dont l'importance a été soulignée. La chasse ritualisée exprime la capacité du roi à contrôler les forces sauvages, dans des espaces - matériellement et symboliquement - interdits aux autres hommes, qui représentent le monde extérieur, le chaos. C'est dans cette perspective qu'a été interprétée la mise en place des forestes par le pouvoir mérovingien au cours du $\mathrm{VII}^{\mathrm{e}}$ siècle ${ }^{22}$. Ces rituels expriment également la capacité du roi à garantir la survie du groupe par l'obtention de subsistances. Or, Reinhard Wenskus constate, dans le cadre du monde germanique, l'existence d'un important parallélisme entre la prise de chasse et le butin pris en guerre ${ }^{23}$. Le mot weida (vieil haut-allemand, à l'origine du mot Beute, le butin) comporte ce double sens. Ce parallélisme suggère que la dimension symbolique du pillage s'inscrit dans le même système

20. R. LE JAN, «La sacralité de la royauté mérovingienne», Annales HSS, 58-6 (2003), p. 1217 1241. Il est probablement réducteur de voir dans ces structures symboliques et en particulier dans la conception guerrière de la royauté un héritage unilatéralement issu du monde germanique, comme le considère par exemple Y. SASSIER, Royauté et idéologie..., p. 73.

21. ÉGINHARD, Vita Karoli, chap. 1, p. 2-4.

22. Cf. J. JARNUT, «Die frühmittelalterliche Jagd unter Rechts- und Sozialgeschichtlichen Aspekten », dans L'uomo di fronte al mondo animale nell'alto medioevo, Settimane del Centro italiano di Studi sull'Alto Medioevo 31, Spolète, 1985, p. 765-798, et les analyses de R. LE JAN, «Le don et le produit sauvage», dans Femmes, pouvoir et société dans le haut Moyen Âge, Paris, 2001, p. 119-131 et EAD., «La sacralité... », p. 1228-1229.

23. R. WensKus, art. «Beute», dans Reallexikon der Germanischen Altertumskunde, vol. II, Berlin, 1976, p. 323-330. 
de représentations. Le roi illustre alors sa force et sa capacité de contrôle des forces chaotiques et sauvages par ses victoires contre les peuples allogènes, et par des pratiques prédatrices qui manifestent son caractère nourricier.

Les récits concernant le butin avar dans l'historiographie carolingienne et, plus généralement, les mentions incessantes de pillages et captures de trésors par des rois, semblent s'expliquer par la permanence de ce système de représentations. Si l'on tient compte des développements en matière d'idéologie royale qui interviennent à partir de la seconde moitié du viII ${ }^{\mathrm{e}}$ siècle, précédemment évoqués, nous parvenons alors à la conclusion que les représentations du pouvoir comportent des éléments hétéroclites qui coexistent. Au-delà d'un aspect purement idéologique, au sens d'un ensemble de conceptions organisées, cohérentes et formalisées, on observe également la permanence de systèmes de valeurs, qui sont plus difficiles à connaître car peu exprimés dans les sources historiographiques ou dans les écrits des érudits du palais, leur transmission relevant principalement de l'oralité. On sait, par le récit d'Éginhard, que Charlemagne fit mettre par écrit des «poèmes anciens et barbares, dans lesquels étaient chantés les guerres et exploits des anciens rois ${ }^{24} »$. Il existe toute une culture orale, qui nous est pour l'essentiel inconnue, mais dont subsistent quelques bribes à travers des poèmes épiques comme le Beowulf ${ }^{25}$. C'est sans doute dans le cadre de cette culture orale et du système de valeurs qu'elle véhicule, que s'exprime la conception d'une royauté guerrière et prédatrice.

L'aristocratie franque demeure largement sensible aux conceptions traditionnelles du pouvoir. Cet aspect a été souligné par Janet Nelson en ce qui concerne la prise du pouvoir par Pépin le Bref en 751. Les sources sur cet événement insistent davantage sur l'élection que sur l'onction, ce qui peut s'expliquer par le fait que le second rituel n'était pas - ou mal - compris par l'aristocratie franque ${ }^{26}$. Or le pouvoir royal, même sous les premiers carolingiens, demeure largement tributaire du soutien de l'aristocratie, ce que Gerd Tellenbach soulignait déjà en évoquant la nécessaire et incessante reconstruction d'un consensus politique entre les rois, les hommes de cour et les grands ${ }^{27}$. La coexistence de modèles idéologiques et symboliques différents, voire contradictoires, peut s'expliquer par la nécessité, pour la royauté carolingienne, de concilier des acteurs variés.

24. ÉGINHARD, Vita Karoli, c. 29, p. 33.

25. Sur la mentalité héroïque, le don d'armes, les relations de fidélité dans le Beowulf, cf. Jos Bazelmans, By Weapons Made Worthy: Lords, Retainers and their Relationship in Beowulf, Amsterdam, 1999.

26. J. L. Nelson, «The Development of Frankish Royal Ritual», dans EAD., Politics and Ritual in Early Medieval Europe, Londres, 1986, p. 292.

27. G. Tellenbach, «Die geistigen und politischen Grundlagen der karolingischen Thronfolge. Zugleich eine Studie über kollektive Willensbildung und kollektives Handeln im neunten Jahrhundert », Frühmittelalterliche Studien, 13 (1979), p. 184-302. 
Bien entendu, cela n'implique pas d'opposer frontalement le haut clergé et les grands laïcs, dès lors qu'ils sont issus des mêmes groupes aristocratiques. Les écrits de certains aristocrates laïques montrent ainsi une convergence idéologique avec les réflexions du clergé ${ }^{28}$. Il n'en demeure pas moins qu'à côté des constructions théoriques sur les fonctions de la royauté, développées par une minorité d'intellectuels, issus principalement du clergé et souvent proches du pouvoir, se maintient un ensemble de représentations qui traversent sans doute assez largement la société carolingienne. Cependant, celles-ci ne se trouvent que rarement explicitées dans les sources, sans doute parce que, comme les croyances pratiques de Pierre Bourdieu, leur force réside précisément dans le fait qu'elles n'ont pas besoin d'être dites ${ }^{29}$. Implicites et donc naturelles, incorporées sans la médiation d'un discours théorique, elles vont de soi, et nous les voyons à l'œuvre à travers les pratiques qui leur sont associées: dans ce cas particulier, par le biais des rituels que les pouvoirs mettent en œuvre autour du butin et de son ostentation.

\section{L'ostentation du butin}

Celle-ci peut prendre plusieurs formes. On peut distinguer, d'une part, des pratiques cérémonielles et, d'autre part, un effort plus général de propagande dont témoignent principalement les sources historiographiques. Les cérémonies et rituels représentent en effet un aspect fondamental de la communication des pouvoirs ${ }^{30}$. Pour remplir son rôle symbolique, le butin doit être vu, ce qui est mis en œuvre par le biais de pratiques ostentatoires. La description la plus précise se trouve dans l'œuvre d'Ermold le Noir, un proche de la cour de Louis le Pieux qui, exilé dans les années 820 , lui consacre un long poème afin de regagner ses faveurs. Dans son récit sur la prise de la ville de Barcelone en 801, il évoque l'envoi, par Louis, d'un convoi au palais d'Aix, avec le butin espagnol:

On amène à Charles, en un long convoi, le butin pris sur les Maures et les présents des chefs: boucliers et cuirasses, vêtements et casques chevelus, un cheval carapaçonné avec un frein d'or. Zado, tremblant et peu joyeux à l'idée de visiter les Francs, figure dans la colonne et traîne la jambe. Le sage Bigon, faisant

28. Cf. par exemple le cas de Dhuoda évoqué par J. Nelson, «Dhuoda», dans P. Wormald et J. Nelson éd., Lay Intellectuals in the Carolingian World, Cambridge, 2007, p. 106-120.

29. P. Bourdieu, Le Sens pratique, Paris, 1980, et en particulier le chapitre $4:$ : La croyance et le corps», p. 111-134.

30. Cf. les études réunies dans F. Theuws et J. L. Nelson éd., Rituals of Power. From Late Antiquity to the Early Middle Ages, Leyde, 2000, ainsi que G. Althoff, Spielregeln der Politik im Mittelalter : Kommunikation in Frieden und Fehde, Darmstadt, 1997 et G. KozIoL, Begging Pardon and Favor : Ritual and Political Order in Early Medieval France, Ithaca, 1991. 
diligence, précède la troupe et arrive le premier à la cour de Charles, porteur de l'heureuse nouvelle. Aussitôt celle-ci se répand dans tout le palais et parvient rapidement aux oreilles de l'empereur. Bigon est appelé, il vient, baise les pieds augustes et s'acquitte de sa mission: «Voici les présents que le fils envoie à son père vénérable, le roi Louis à l'empereur Charles: il les a conquis de haute lutte sur les Maures, en personne, épée et bouclier au poing. Il vous fait amener, en outre, le roi de la ville, qu'il a pris de vive force: voici Zado devant César. La ville qui jadis a nui à tant de Francs est terrassée: elle a capitulé ${ }^{31}$.»

Ce récit imagé suggère l'existence d'une véritable mise en scène qui a pour objet d'illustrer les richesses envoyées et la victoire de Louis. Dans le cas des Avars, on ne connaît aucun récit aussi circonstancié sur le transport du butin, mais les récits suggèrent que les membres de la cour carolingienne furent fortement impressionnés par son arrivée. Le récit des Annales royales, par exemple, est construit sur l'arrivée du butin à Aix, plutôt que sur les expéditions elles-mêmes ${ }^{32}$. Tout suggère que le trésor fut amené à Aix-la-Chapelle en grande pompe. Les Annales nordhumbrani mentionnent l'envoi de quinze chariots, tirés par quatre bœufs chacun, chargés en or, argent, soieries ${ }^{33}$.

Une autre pratique qu'il faut envisager ici est celle de la distribution du butin. Si on considère en effet que le butin doit être vu, les dons qu'il permet de faire représentent une occasion privilégiée de le montrer. L'arrivée du butin avar est suivie d'une distribution mentionnée par plusieurs sources. Charles en donne une partie aux grands, clercs et laïcs, ainsi qu'à ses fidèles, sans doute les vassaux royaux ${ }^{34}$. Cet acte est probablement mis en œuvre dans le cadre de

31. ERmold le Noir, Poème en honneur de Louis le Pieux, v. 572-598 : «Ducitur interea ad Carolum longo ordine praeda / Maurorum spoliis muneribusque ducum, / Arma et loricae, vestes galeaeque comantes, / Partus equus faleris, aurea frena simul. / Zado tremens nimium, nolensque revisere Francos, / Pergit et ipse pigro consociante gradu. / Bigo catus properans antevolat agmen, et aulam / Primus adest Caroli, nuntia laeta ferens. / Fama recens totam commiscuit ocius aulam, / Caesareas aures mox penetravit ovans. / Bigo vocatus adest, plantis dat basia celsis, / Et sequitur verbis ordine iussa sibi: / «Filius ecce pio transmittit munera patri, / Augusto Carolo rex Hludowicus enim, / Munera, quae gladio, scuto, proprioque lacerto / Extulit a Mauris victor habenda sibi. / Regem etiam vobis urbis, quem cepit in armis, / Misit, adest Zadun Caesaris ante oculos. / Urbs, quae Francorum multos populaverat olim, / Victa iacet bello, regia iussa petens. » (éd. et trad. E. FARAL, Paris, 1964, p. 46-48).

32. Cf. supra, note 5 .

33. Cf. supra, note 14.

34. Cf. Annales Regni Francorum, a. 796, p. $98: « \ldots$ porro reliquam partem obtimatibus, clericis sive laicis, ceterisque fidelibus suis largitus est.»; Annales qui dic. Einhardi, a. 796, éd. F. KuRzE, MGH SS Rer. Germ. in us. sch. 6, Hanovre, 1895, p. 99: «... reliquum vero inter optimates et aulicos ceterosque in palatio suo militantes liberali manu distribuit.» Annales Laureshamenses, a. 795, p. 36 : $\ll \ldots$ et distribuit ipsum thesaurum inter ecclesias et episcopos, seu abbates et comites; nec non et universos fideles suos de eodem thesauro mirifice honoravit.» 
pratiques cérémonielles de cour ${ }^{35}$. Il s'inscrit dans la logique de l'échange de dons et, en tant que tel, représente un facteur de renforcement et de hiérarchisation des liens sociaux au niveau des élites, à une époque où le pouvoir carolingien s'efforce précisément d'accentuer les liens de fidélité comme élément de cohésion du royaume.

Cela permet de préciser la place du butin comme facteur de légitimation du pouvoir royal. Le caractère prédateur de la royauté ne se justifie que dans la mesure où il s'inscrit dans une logique de redistribution, ce qui est également valable, de manière plus générale, en ce qui concerne le rapport des élites à la richesse, dont la légitimité, associée à l'idée de largitas, repose sur la réinsertion des biens accumulés dans des circuits de distribution, autrement dit, sur leur mobilisation dans le cadre de l'échange social ${ }^{36}$. Dans des sociétés aussi marquées par le fait guerrier, il est vraisemblable que les objets du butin constituent un élément privilégié dans les échanges au sein des élites. Si l'on considère que les échanges de dons ont pour caractéristique, essentiellement, de structurer du lien social, l'origine étrangère des objets du don inscrit ce processus dans une logique de distinction, de différentiation par rapport à l'autre et comporte alors également une dimension identitaire. La distribution du butin avar par Charlemagne aux élites du royaume permet non seulement de manifester et renforcer des liens de fidélité particuliers, mais également d'illustrer la cohésion du peuple franc autour de son roi.

D'autres acteurs bénéficient de ces largesses. Dès la réception du trésor, Charlemagne fait don au roi Offa de Mercie d'un baudrier et d'une épée avars ${ }^{37}$. Cet envoi s'inscrit dans le cadre classique des dons diplomatiques, mais il est

35. Il reste à déterminer dans quelle mesure cela s'inscrit dans la continuité de pratiques traditionnelles. Dans le monde franc à l'époque mérovingienne, le butin semble plutôt faire l'objet d'un partage égalitaire, sur le modèle du récit de Grégoire de Tours sur le vase de Soissons (GRÉGOIRE DE Tours, Historiarum decem libri, II, 27, éd. B. KRUSCH, MGH SRM 1.1, Hanovre, 1951, p. 71-73). La distribution par le chef s'inscrit plutôt dans la logique de la Gefolgschaft de Tacite et de ses avatars (antrustionat franc). La question doit encore être approfondie. En 732, après la bataille de Poitiers, les guerriers de Charles Martel se partagent le butin ( Spolias tantum/Et manubias inter se divisas » : ANonyme de CoRdoue, Chronique rimée des derniers rois de Tolède, éd. P. J. Tailhan, 1885 , p. 40). En revanche, en 774, après la prise de Pavie, Charlemagne distribue une partie du butin aux siens («thesauros... dedit exercitui suo» : Annales Laurissenses minores, a. 775, éd. G. PERTZ, MGH SS 1, p. 117). Timothy Reuter («Plunder...»,p. 79-80) y voit une évolution et estime que les Carolingiens parviennent à exercer un contrôle plus étroit sur le produit des pillages.

36. Cf. à ce sujet le récent colloque de Bruxelles (mars 2008) : J.-P. Devroey, L. Feller et R. Le JAN, éd. Les Élites et la richesse au haut Moyen Âge, Turnhout, 2010.

37. AlCUINI epistolae, $\mathrm{n}^{\circ} 100$, p. 144: «Vestrae quoque dilectioni ad gaudium et gratiarum actiones Deo omnipotenti dirigere studuimus unum balteum et unum gladium Huniscum et duo pallia sirica... ». Sur le contexte de ce don, cf. J. WALLACE-Hadrill, «Charlemagne and England», dans ID., Early Medieval History, Oxford, 1975, p. 164-166. 
significatif que la lettre d'Alcuin tienne à préciser qu'il s'agit là d'armes avares, ce qui suggère une logique ostentatoire et implique probablement une dimension agonistique, en raison de la démonstration de puissance que ce don permet. Parmi les bénéficiaires de ce butin nous trouvons, d'après les Annales de Lorsch, également les églises, ce qui va dans le sens du vœu exprimé par Théodulphe d'Orléans. Ce clerc, éminent savant de la cour carolingienne, et proche conseiller de Charles, incite ce dernier à rendre grâce au Seigneur pour les richesses prises en Pannonie, dans un de ses nombreux poèmes ${ }^{38}$. Enfin, des dons sont faits au pape Léon III, par l'intermédiaire d'Angilbert, abbé de Saint-Riquier ${ }^{39}$. Celui-ci apporte avec lui également une célèbre lettre, rédigée par Alcuin, qui exprime la position carolingienne concernant les pouvoirs respectifs du roi franc et du pape : «À moi il appartient, avec l'aide de la divine piété, de défendre en tout lieu la sainte Église du Christ par les armes : au dehors contre les incursions des païens et les dévastations des infidèles; au-dedans en la protégeant par la diffusion de la foi catholique ${ }^{40}$.» Le don d'objets provenant du butin vient ici appuyer une revendication politique qui s'inscrit dans une réflexion plus générale sur la nature du pouvoir royal et sur l'identification entre royaume des Francs et populus Christianorum, dont le pouvoir carolingien se proclame le défenseur, ce qui est un aspect central de l'idée impériale alors en développement.

La prédation de richesses fait également l'objet d'une propagande dont témoignent les sources historiographiques. Le grand nombre de textes évoquant le pillage du trésor avar, aussi bien proches du pouvoir que plus lointains, illustre la force de propagation de la nouvelle et suggère l'existence d'un effort délibéré mené dans ce sens par la cour carolingienne. L'impact fut tel que l'événement a pris un caractère légendaire au cours du $\mathrm{IX}^{\mathrm{e}}$ siècle. Le récit de Notker le Bègue est révélateur à cet égard. Ce moine et lettré de l'abbaye de Saint-Gall écrit, dans les années 880, un recueil d'histoires édifiantes sur Charlemagne, les Gesta Karoli. Il y décrit le hring avar comme une structure fortifiée à neuf cercles, le plus large de ces cercles ayant un diamètre correspondant à la distance entre Constance et Zürich (environ $60 \mathrm{~km}$ ). Ensuite, il évoque la conquête de Charlemagne et la

38. THEODULFI carmina, Carmen 25, v. 33-36: «Percipe multiplices laetanti pectore gazas, / Quas tibi Pannonico mittit ab orbe Deus. / Inde pias celso grates persolve tonanti, / Cui, solet ut semper, sit tua larga manus.» (éd. E. DüMmLer, MGH Poet. Lat. aevi Carol. 1, p. 485).

39. Annales Regni Francorum, a. 796, p. 98: «Quo accepto peracta Deo largitori omnium bonorum gratiarum actione idem vir prudentissimus atque largissimus et Dei dispensator magnam inde partem Romam ad limina apostolorum misit per Angilbertum dilectum abbatem suum...». D'après P. DeLOGU, «Oro e argento in Roma tra il vil e il Ix secolo», dans Cultura e società nell'Italia medievale. Studi per Paolo Brezzi, vol. I, Rome, 1988, p. 273-293, l'augmentation des mentions d'or et d'argent dans le Liber pontificalis s'expliquerait principalement par cette donation.

40. AlCUINI epistolae, $\mathrm{n}^{\circ}$ 93, p. 136-138. 
distribution du trésor ${ }^{41}$. Ainsi que le souligne Walter Pohl à juste titre, ce récit comporte un fort caractère légendaire. Cependant, il est possible que la description du hring avar par Notker résulte d'une distorsion de données historiques précises : on connaît l'existence, dans certaines sociétés turcophones d'Asie centrale, de vastes camps circulaires de tentes, dans lesquels sont installés les khagans. Ces camps sont sommairement fortifiés et eux-mêmes parfois entourés par un second cercle de structures où résident les dignitaires proches du pouvoir. Le centre du pouvoir avar correspondait probablement à une structure circulaire de ce type ${ }^{42}$.

Notker est le dépositaire d'une longue tradition se transmettant oralement. Dans son récit, il précise que les informations lui viennent d'un vieil homme, un certain Adalbert, qui l'a élevé dans sa jeunesse. Ce dernier aurait participé aux campagnes avares, sous les ordres du praefectus de Bavière Gerold ${ }^{43}$. Dans les années 790, lors de la campagne militaire contre les Avars, un ensemble de rumeurs concernant ce peuple existait déjà, comme en témoignent les sources évoquant les trésors du hring. Les Annales royales mentionnent des richesses accumulées «pendant de nombreux siècles » par les khagans ${ }^{44}$. D'ailleurs, l'utilisation, par les annalistes francs, du terme hring, un mot qui signifie, en vieil hautallemand, «anneau», «cercle » ${ }^{45}$, pour désigner la fortification avare, illustre bien le caractère fragmentaire et approximatif des connaissances que les Francs avaient de ce peuple. L'anecdote de Notker s'inscrit dans la continuité de cet ensemble de connaissances en parties réalistes, en partie légendaires. Son témoignage montre non seulement que les récits sur les Avars gardaient toute leur force au cours du $\mathrm{IX}^{\mathrm{e}}$ siècle, mais également que cet ensemble de représentations s'était enrichi des événements des années 790, ce qui témoigne a fortiori de leur écho, et du prestige qui pouvait auréoler ceux qui s'étaient emparés d'un tel trésor.

\section{Un enjeu de la compétition entre les élites}

Comme on l'a vu, en 801, Louis le Pieux envoie à son père Charles un convoi avec une part du butin prélevé à Barcelone. On trouve de manière relativement fréquente des cas de présentation du butin par des grands ou des guerriers à leur supérieur. Le terme «présentation» est pleinement pertinent, employé par les sources elles-mêmes : en 796, d'après la réécriture des Annales

41. Notker le Bègue, Gesta Karoli magni imperatoris, II, 1, éd. R. Rau, Quellen zur karolingischen Reichsgeschichte, vol. III, Darmstadt, 1960, p. 376-378.

42. W. Pohl, Die Awaren..., p. 307-308 et ID., «The regia and the hring - barbarian places of power», dans M. DE Jong, F. Theuws et C. van RhiJn éd., Topographies of Power in the Early Middle Ages, Leyde, 2001, p. 439-461.

43. NotKer le Bègue, Gesta Karoli, I, 34 et II, 1, p. 374 et 376.

44. Cf. supra, note 5 .

45. R. SchütZeichel, Althochdeutsches Wörterbuch, Tübingen, 1995 (1969), p. 239. 
royales des années 810 , Pépin se rendit à Aix et «praesentavit» à Charles les «dépouilles du royaume ${ }^{46}$ ». La pratique semble alors habituelle. En 798, peu de temps après Pépin, Alphonse d'Asturie envoie à Aix le butin et les captifs qu'il a pris aux Sarrasins lors de la conquête de Lisbonne ${ }^{47}$. Les objets envoyés sont qualifiés d'insignia victoriae. Le butin remplit alors une fonction de trophée. Dans certains cas, c'est un véritable trophée qui est remis au chef. En 799, les Sarrasins attaquent les Baléares mais sont défaits par les Francs: ce sont alors leurs insignes (signa Maurorum) qui sont présentées à Charles ${ }^{48}$. La même année, Gui, en charge de la marche de Bretagne, pénètre dans le domaine breton. Après sa victoire, il présente au roi les armes des grands vaincus, sur lesquelles étaient inscrits leurs noms, et qui avaient été données en signe de reddition ${ }^{49}$. Plus tardivement, en 865, Robert le Fort, après sa victoire contre les Normands, envoie leurs étendards et leurs armes à Charles le Chauve ${ }^{50}$. Dans d'autres cas, l'information peut être transmise par voie de correspondance. En 869, Carloman, fils de Louis le Germanique, mène une campagne contre Rastiz, dux des Bohémiens. Il s'empare alors d'un grand butin, et en informe son père par une lettre ${ }^{51}$.

Ces pratiques de présentation du butin par les grands sont introduites pendant les années 790 et tendent rapidement à se multiplier. D'après Michael McCormick, cela pourrait s'expliquer par le nombre croissant d'expéditions militaires menées par d'autres personnalités que le roi en personne ${ }^{52}$. Elles semblent reprendre un modèle byzantin. Ce n'est peut-être pas un hasard qu'Éric, duc de Frioul, qui a l'initiative du premier cas attesté de ces pratiques, autrement dit l'envoi du trésor avar en 795, fût également en charge de l'Istrie, enclave byzantine sur la côte dalmate, qui avait été conquise par les Francs en 788. On peut suggérer que c'est par ce canal que la coutume a été reprise et introduite par Éric.

Ces pratiques ostentatoires correspondent clairement à des stratégies de mise en valeur et de ce point de vue, elles sont susceptibles de constituer un

46. Annales qui dic. Einhardi, a. 796, p. 99.

47. Annales Regni Francorum, a. 798, p. 104.

48. Annales Regni Francorum, a. 799, p. 108: «Signa quoque Maurorum in pugna sublata et domno regi praesentata sunt.»

49. Annales Regni Francorum, a. 799, p. 108: «et regi [..]. arma ducum, qui se dediderant, inscriptis singulorum nominibus praesentavit.»

50. Annales Bertiniani, a. 865, éd. G. WAITz, MGH SS Rer. Germ. in us. sch. 5, Hanovre, 1883, p. 79: «Rodbertus... vexilla et arma Nortmannica Karolo mittit.»

51. Annales Fuldenses, a. 869, éd. F. KuRze et G. H. PerTz, MGH SS Rer. Germ. in us. sch. 7 , Hanovre, 1891, p. 67: «... praedam inde capiens non modicam sicut ipse litteris ad patrem suum destinatis retulit.»

52. M. МсСовміск, Eternal victory. Triumphal Rulership in Late Antiquity, Byzantium, and the Early Medieval West, Cambridge, 1986, p. 375-376. 
aspect de la compétition entre les élites. S'il est difficile d'établir clairement une corrélation entre les pratiques ostentatoires et l'obtention d'honores, domaines fonciers, richesses, positions privilégiées, quelques éléments vont toutefois dans ce sens. La source la plus explicite à ce propos est un diplôme de Charlemagne, rédigé en mars 795 et concernant un certain Jean, qui a combattu contre les Sarrasins pour le compte de Louis le Pieux. Le texte nous apprend qu'après des combats ayant eu lieu dans le pagus Barchinonense, Jean a apporté à Louis le Pieux une part du butin, comprenant une broigne, une épée, et un écrin. Après lui avoir présenté ce butin, il lui a demandé un domaine dans la région de Narbonne ${ }^{53}$. Louis le Pieux a accepté et l'a envoyé à Charles avec une lettre écrite de sa main, pour que Jean se recommande à Charles et pour que ce dernier lui confirme la donation. Les pratiques de présentation du butin favorisent donc la mobilité sociale, dans le cas présent en permettant l'obtention de terres, et sont explicitement mises en œuvre à cet effet. Par extension, il semble extrêmement probable qu'au niveau de la strate supérieure des élites, les pratiques ostentatoires du butin s'inscrivent dans des stratégies de valorisation qui favorisent l'obtention de charges et avantages divers.

Le cas du pillage des Avars semble précisément correspondre à une démarche de type compétitif. L'expédition lancée à l'automne 795 par Éric de Frioul résulte clairement d'une initiative personnelle, n'étant pas commanditée par le pouvoir central. Les Avars connaissaient alors une période de faiblesse interne, en raison d'un conflit civil et de la mort du khagan. Éric de Frioul se trouvait être le représentant carolingien le plus proche géographiquement du centre de l'empire avar. Il est probable qu'il est le premier informé de la situation et qu'il décide alors de mettre à profit la situation pour s'emparer du mythique trésor des Avars. Cela correspond d'ailleurs assez bien à un schéma observable dans d'autres contextes, les élites carolingiennes installées aux frontières ayant tendance à mener une politique propre, parfois en contradiction avec les orientations du pouvoir royal ${ }^{54}$. La possibilité d'attaquer le noyau avar sans concertation préalable avec le pouvoir central représentait sans doute une occasion de se mettre en avant, par l'envoi du trésor à la cour carolingienne, pratique dont on a vu qu'Éric a peut-être précisément été l'initiateur.

53. Diplôme nº 179, éd. E. MüHlbacher, MGH Diplomata Karolinorum 1, Hanovre, 1906, p. 241-242.

54. Cela s'observe tout particulièrement dans le contexte des relations des pouvoirs frontaliers orientaux avec les populations slaves situées au-delà de l'Elbe et de la Saale. Cf. T. LiENHARD, «Les chiens de Dieu». La politique slave des Mérovingiens et des Carolingiens, thèse de doctorat sous la dir. de R. LE JAN, Lille, 2003, p. 297-415 et ID., «À qui profitent les guerres en Orient? Quelques observations à propos des conflits entre Slaves et Francs au IX siècle», Médiévales, 51 (2006), p. 69-83. 
La seconde expédition, lancée par Pépin d'Italie à l'été 796, donne l'impression d'être une expédition de rattrapage. Elle est menée sans Éric de Frioul, qui n'est pas mentionné dans les récits, ce qui est étonnant, si on considère sa position frontalière. Les sources signalent en revanche l'envoi par Charlemagne de forces bavaroises et alémaniques en renfort à Pépin. Pendant son avancée, avant même son arrivée au hring, le khagan se rend sur le Danube où stationne Pépin pour se soumettre. Cela n'empêche pas ce dernier de poursuivre sur sa lancée, de détruire la fortification avare et de s'emparer de ce qu'il reste du trésor. Il faut noter que le trésor pris par Pépin est transporté à Aix par deux convois successifs. Une première part est envoyée alors qu'il demeure sur place. Ensuite, il amène lui-même à Aix ce qui reste du butin, ce qui contribue sans doute à son entrée triomphale ${ }^{55}$. Pépin, sans doute, ne pouvait faire moins bien qu'Éric et souhaitait obtenir une part du prestige qui rejaillirait inévitablement sur celui qui parviendrait à s'emparer des légendaires richesses avares. Il ne s'agit là, bien sûr, que d'une interprétation des événements : rien ne permet d'affirmer que les enjeux se posaient en ces termes, mais les éléments allant dans ce sens sont nombreux.

Quelques éléments postérieurs confortent cette interprétation. Certaines sources donnent au récit des événements une inflexion par laquelle elles soulignent le rôle de Pépin. C'est le cas du texte connu sous le nom d'Historia Langobardorum codicis Gothani, qui correspond à un remaniement de l'Origo gentis Langobardorum (récit d'origine des Lombards du VII siècle), opéré au début du $\mathrm{IX}^{\mathrm{e}}$ siècle par un auteur anonyme, dans une perspective chrétienne et carolingienne ${ }^{56}$. L'auteur de ce récit évoque uniquement le pillage de Pépin, qu'il présente comme une juste récupération d'objets liturgiques volés aux églises par les Avars ${ }^{57}$. Éginhard, dans sa Vita Karoli, de la même manière, n'évoque que l'expédition de Pépin. Éric est mentionné dans son récit, mais uniquement pour

55. Le récit le plus complet sur l'expédition de Pépin est celui des Annales Laureshamenses, a. 796, p. 36: «In ipso aestate transmisit rex Carolus Pippinum filium suum cum suis quos in Italia secum habebat, et Paioarios cum aliqua parte Alamaniae in finibus Avarorum; et coadunatus est Pippinus cum omnibus quos pater eius ei transmisit in solacium, et transito Danovio, cum exercitu suo pervenit ad locum, ubi reges Avarorum cum principibus suis sedere consueti erant, quem et in nostra lingua Hringe nominant; et inde tulit thesauros multiplices et transmisit patri suo, et ipse postea cum exercitu suo et magnis thesauris Avarorum pervenit in Francia.» Il doit être complété par celui des Annales royales ainsi que par leur réécriture postérieure attribuée à Éginhard: cf. supra, note 5 et 45 .

56. Le récit fut publié sous ce titre au XIX ${ }^{\mathrm{e}}$ siècle: Historia Langobardorum codicis Gothani, éd. G. WAITZ, MGH SS Rer. Langob., Hanovre, 1878, p. 7-11. Il est ainsi appelé car il n'est connu que par un manuscrit de Gotha (Forschungsbibliothek Gotha, Memb. I 84). Sur ce texte, cf. M. CoumerT, Origines des peuples. Les récits du haut Moyen Âge occidental (550-850), Paris, 2007, p. 251-261.

57. Historia Langobardorum, p. 11: «... multa vasa sanctorum, quae illi crudeles et impii rapuerunt, per istum defensatorem sunt ad propriam reversa.» 
en signaler la mort, en 799, lors de nouveaux combats contre les Avars ${ }^{58}$. Quant à Éric de Frioul, il est significatif que son nom apparaisse pour la première fois précisément dans les récits annalistiques concernant les faits de 795. Alcuin luimême lui adresse alors une lettre, dans laquelle il le félicite pour sa victoire ${ }^{59}$. À l'évidence, cet acte permet à Éric de figurer parmi les personnages ayant marqué l'année 795, de renforcer sa position à la cour carolingienne et d'approfondir ses relations personnelles avec l'entourage le plus proche du roi franc.

La documentation carolingienne permet ainsi d'entrevoir les multiples enjeux que comporte le butin, envisagé à la lumière des conceptions propres à la société franque, comme son importance dans la représentation du pouvoir. La guerre est une réalité majeure de la société carolingienne et, s'il est vrai que l'entourage royal s'efforce, au tournant du $\mathrm{VIII}^{\mathrm{e}}$ au $\mathrm{IX}^{\mathrm{e}}$ siècle, de développer un apparat idéologique autour de la notion de «guerre juste», visant à légitimer l'expansion carolingienne, les pratiques et les rituels mis en œuvre révèlent la permanence de systèmes de représentations qui valorisent l'exploit militaire et le butin en tant que tels, lesquels constituent alors des moyens d'affirmer les hiérarchies et de conforter les dominations. Les stratégies de représentation du pouvoir royal s'articulent alors sur des registres différents, alors que les grands rehaussent leur prestige à la faveur de leurs exploits personnels, obéissant à une logique compétitive qui est centrale dans les dynamiques sociales aristocratiques.

Bien sûr, il ne s'agit pas là que d'un apparat symbolique. Celui-ci n'est qu'un aspect de l'«économie du pillage, du don et de la largesse» évoquée par Georges Duby, laquelle continue d'organiser une large part de la circulation des richesses dans la société franque ${ }^{60}$. Pour Timothy Reuter, son importance est telle que le tarissement des revenus lié à la fin de l'expansion carolingienne constituerait un facteur déterminant de l'instabilité politique croissante de la première moitié du ${ } X^{\mathrm{e}}$ siècle dans le monde franc ${ }^{61}$. C'est précisément à ce moment qu'apparaissent de nouveaux circuits de la prédation, au bénéfice des Vikings nouvellement apparus, mais également de la Francie orientale de Louis le Germanique, dont la stabilité politique repose précisément, entre autre, sur les richesses considérables tirées des tributs et pillages des domaines slaves orientaux ${ }^{62}$.

Rodolphe KelLer - Université Paris-Est, Laboratoire ACP, EA 3350, 5 boulevard Descartes, Champs-sur-Marne, F-77454 Marne-la-Vallée Cedex 2

58. ÉGINHARD, Vita Karoli, c. 13, p. 16.

59. AlCUINI epistolae, $\mathrm{n}^{\circ} 98$, p. 142.

60. G. Duby, Guerriers et paysans..., p. 69.

61. T. Reuter, «The end of Carolingian military Expansion», dans P. Godman et R. Collins éd., Charlemagne's Heir: New Perspectives on the Reign of Louis the Pious (814-840), Oxford, 1990, p. 391-405.

62. ID., «Plunder...», p. 75-94. 


\section{Pillages et butins dans la représentation du pouvoir à l'époque carolingienne}

La documentation d'époque carolingienne témoigne de l'importance du butin comme élément de représentation du pouvoir. À la charnière des VIII et IX ${ }^{\mathrm{e}}$ siècles, le royaume franc atteint son extension maximale, et les intellectuels carolingiens développent alors un apparat idéologique sur la notion de «guerre juste», visant à légitimer l'expansion franque. Cependant, l'étude des pratiques ostentatoires montre que la légitimité royale continue de reposer sur des systèmes de valeurs qui dépassent le cadre idéologique et qui valorisent l'exploit militaire ou la conquête d'un riche butin en tant que tels.

La prise du butin avar en 795/796 ne constitue qu'un exemple particulièrement frappant des enjeux que soulève la prédation de richesses. L'historiographie carolingienne témoigne de l'effort mené afin de faire connaître l'immensité du trésor avar. Sa distribution remplit des fonctions aussi bien sociales, que politiques et diplomatiques. Elle manifeste la puissance militaire du roi, tout en renforçant les liens de fidélité avec les grands, bénéficiaires d'une part des richesses, parmi d'autres acteurs. Les grands eux-mêmes rivalisent entre eux, en apportant au roi de riches butins, selon une logique compétitive déterminante dans les dynamiques de hiérarchisation des élites.

Carolingiens - guerre - butin - Avars - élites - pouvoir - idéologie - pillage.

\section{Plunder and Booties in the Carolingian's Representation of Power}

The Carolingian documentation gives us an interesting insight into the place of the booty as element of political legitimacy. While the Frankish kingdom is reaching its largest extension, Carolingian scholars build up an ideological framework, in order to legitimate the military expansion with the notion of «right war». Nonetheless, we observe several practices which give evidences of the persistance of traditional royal conceptions, based upon bare military performance and conquest of rich booties.

The conquest of the Avar booty in 795/796 is a striking example of the multiplicity of practices related to predation. Carolingian historiography shows a real political effort to publicize the greatness of the Avar threasure. Its distribution takes place as a ritual permitting the reinforcement of links between the king and the magnates, which benefit of this wealth, among other actors, like the churches or the pope. The symbolic role of the booty also has an impact on aristocratic conducts. By sending parts of spoils to the king, as trophees, the magnates publicly demonstrate their military virtue. As such, those practices are part of the competitive strategies which contribute to the continual reshaping of social hierarchies.

Carolingians - war - booty - Avars - elites - power - ideology - plunder. 